\title{
Landscape structure and genetic architecture jointly impact rates of niche evolution
}

\author{
Katja Schiffers, Frank M. Schurr, Justin M. J. Travis, Anne Duputié, Vincent M. Eckhart, \\ Sébastien Lavergne, Greg Mclnerny, Kara A. Moore, Peter B. Pearman, Wilfried Thuiller, \\ Rafael O. Wüest and Robert D. Holt
}

K. Schiffers (katja.schiffers@gmail.com), S. Lavergne, W. Thuiller and R. O. Wüest, Univ. Grenoble Alpes, LECA, FR-38000 Grenoble, France, and CNRS, LECA, FR-38000 Grenoble, France. - P. B. Pearman and ROW, Landscape Dynamics Unit, Swiss Federal Research Inst. WSL, 111 Zürcherstraße, CH-8903 Birmensdorf, Switzerland. - F. M. Schurr, Inst. des Sciences de l'Evolution de Montpellier, UMR-CNRS 5554, Univ. Montpellier II, FR-34095 Montpellier cedex 5, France, and Inst. of Landscape and Plant Ecology, Univ. of Hohenheim, DE-70593 Stuttgart, Germany. - J. M. J. Travis, Inst. of Biological and Environmental Sciences, Univ. of Aberdeen, Zoology Building, Tillydrone Avenue, Aberdeen, AB242TZ, UK. - A. Duputié, Laboratoire Génétique et Évolution des Populations Végétales, UMR CNRS 8198, Univ. des Sciences et Technologies de Lille 1, FR-59655 Villeneuve d'Ascq Cedex, France. - V. M. Eckhart, Dept of Biology, Grinnell College, Grinnell, IA, USA. - G. McInerny, Dept of Computer Science, Univ. of Oxford, Wolfson Building, Parks Road, OX1 3QD, UK, and Computational Science Laboratory, Microsoft Research Ltd, 21 Station Road, Cambridge, CB1 2FB, UK. - K. A. Moore, Dept of Evolution and Ecology, Univ. of California, Davis, CA 95616, USA. - R. D. Holt, Dept of Biology, Univ. of Florida, Gainesville, FL 32605, USA.

\begin{abstract}
Evolutionary adaptation is a key driver of species' range dynamics. Understanding the factors that affect rates of adaptation at range margins is thus crucial for interpreting and predicting changes in species' ranges. The spatial structure of environmental conditions is one of the determinants of whether and how quickly adaptations occur. However, while landscape structures at range edges are typically complex, most theoretical work has so far focused on relatively simple environmental geometries.

Using an individual-based allelic model, we explore the effects of different landscape structures on the rate of adaptation to novel environments and investigate how these structures interact with the genetic architecture of the trait governing adaptation and the dispersal capacity of the considered species. Generally, we find that rapid adaptation is favored by a good match between the coarseness of the trait's genetic architecture (many loci of small effects versus few loci of large effects) and the coarseness of the landscape (abruptness of transitions in environmental conditions). For example, in rugged landscapes, adaptation is quicker for genetic architectures with few loci of large effects, while for shallow gradients the opposite is true. Moreover, dispersal capacities affect the rate of adaptation by modulating the 'apparent coarseness' of the landscape: a gradient perceived as smooth by species with limited dispersal capacities appears rather steep for highly dispersive ones. We also find that the distribution of evolving phenotypes strongly depends on the interplay of landscape structure and dispersal capacities, ranging from two distinct phenotypes for most rugged landscapes, over the co-occurrence of an additional third phenotype for highly dispersive species, to the whole range of phenotypes on smooth gradients.

By identifying basic factors that drive the fixation probability of newly arising beneficial mutations, we hope to further broaden the understanding of evolutionary adaptation at range margins and, hence, species' range dynamics.
\end{abstract}

Understanding the factors that govern the distribution and dynamics of species ranges is a fundamental objective of ecology and biogeography. The ecological niche of a species (Hutchinson 1978, Holt 2009) characterizes those combinations of biotic and abiotic environmental conditions that permit populations of that species to persist, owing to how its key traits determine the balance of birth and death rates. To a first approximation, and in the absence of evolution and

This is an open access article under the terms of the Creative Commons Attribution License, which permits use, distribution and reproduction in any medium, provided the original work is properly cited. source-sink effects (Pulliam 2000), a species' range that has reached equilibrium can be viewed as a projection of that species' niche in geographic space (Soberón 2007, Sexton et al. 2009). Yet, evolution proceeds unceasingly to shape populations' adaptation to the biotic and abiotic environment (Lavergne et al. 2010). This is well illustrated by the fact that $45-70 \%$ of natural plant populations appear to be locally adapted, i.e. local individuals perform better at their home sites than do transplanted foreign ones (Leimu and Fischer 2008, Fournier-Level et al. 2011). Species ranges thus reflect a mosaic of adaptations to varying environments, and static geographic limits are not necessarily expected. In consequence, a full understanding of species' range dynam- 
ics is only possible in the light of evolutionary adaptation and constraints on such adaptation.

At range limits, where dispersal can readily expose individuals to conditions falling outside the species' niche, natural selection is particularly strong and may trigger adaptation to new habitats and, thus, niche evolution and subsequent expansion of the species' geographical range (Levins 1968, Holt and Gaines 1992, Kawecki 2008). Whether and how quickly adaptation occurs is governed by a number of demographic and evolutionary factors, notably by population size, the additive genetic variance and covariance of the traits under selection, mutation rate, and the probability of fixation of beneficial mutations (Bürger 2000). The probability of fixation for a given mutation depends in turn on how selection affects population demography (the so-called 'costs of natural selection', Haldane 1957), how demography influences selection (e.g. density-dependence), and how closely the resulting phenotype matches the local phenotypic optimum. Moreover, because organisms disperse, they can experience a range of conditions with varying selective optima in spatially heterogeneous environments. The process of adaptation should therefore also be influenced by the spatial structure of the biotic and abiotic environment, as the geometry of conditions defines how the phenotypic optimum varies across space (Siepielski et al. 2013).

To date, most theoretical work on adaptive evolution at range limits has explored relatively simple environmental geometries, such as smooth gradients (Kirkpatrick and Barton 1997) and coupled discrete habitats (e.g. source-sink models, Holt and Gaines 1992, Kawecki 1995, 2008, Ronce and Kirkpatrick 2001). Yet, landscape structure at range limits typically exhibits more complex patterns than are commonly assumed by theoretical models of adaptation (Fortin et al. 2005, Gastner et al. 2009). Prior studies have shown the importance of these structures for the dynamics of adaptation (Lieberman et al. 2005, Holt and Barfield 2011, Frean et al. 2013). For example, starting with simple two-patch source-sink models and stabilizing selection on a single trait, with different optima in the source and sink, Holt and Barfield (2011) showed that the addition of another occupied patch with a different selection optima can strongly alter the rate at which adaptation to a focal sink patch takes place. Also, Lieberman et al. (2005) demonstrated that spatial structuring of dispersal could determine evolutionary outcomes by suppressing or amplifying the strength of selection. Still, little work has been conducted to compare effects of common and more realistic landscape geometries, such as patches of suitable habitat embedded within a matrix of unsuitable conditions (Roy and Thomas 2003, Kunin et al. 2009), on the pace of adaptation at species range limits (Holt and Barfield, 2011, Siepielski et al. 2013).

In addition to landscape structure, the genetic architecture of traits under selection may influence the likelihood and rate of adaptation to novel conditions (Kawecki 2000, 2008, Kimbrell and Holt 2007, Gomulkiewicz et al. 2010, Kimbrell 2010). A so far unresolved question is whether adaptation in polygenic traits (which typically are believed to be those involved in adaptation to marginal environments) is more likely to arise as a result of many mutations of small phenotypic effects or as a result of a few large mutations (Orr 2005). While the conventional wisdom in evolutionary biology for many years was that most adaptations reflect the buildup of beneficial mutations that have rather small phenotypic effects (Fisher 1930), in some cases, mutations with large effect on fitness are required for adaptation to novel conditions (Holt and Gomulkiewicz 1997, Orr 2005). For example, adaptation to insecticides in mosquitoes was shown to depend upon rare alleles at just a few loci that propagated a large phenotypic effect (Bourguet et al. 2004). Also in their three-patch model, Holt and Barfield (2011) found that adaptation was eventually driven by a few but large mutations, even though initially genetic variation in the trait under selection was dependent on many alleles of small effect.

Finally, dispersal is a key evolutionary factor, modulating the probability and rate of adaptation to new environmental conditions by a number of mechanisms. Dispersal may, for instance, facilitate adaptation by spreading beneficial alleles throughout a population's range (Davis and Shaw 2001, Bell and Gonzalez 2011) or hamper adaptation by promoting gene swamping (Garcia-Ramos and Kirkpatrick 1997, Bridle and Vines 2007). An interactive effect may arise among the dispersal capacity of a species, its genetic architecture, and the structure of the landscape: dispersal characteristics and the structure of the selective landscape jointly determine the difference in habitat conditions to which a dispersing individual is exposed. The magnitude of this difference in turn defines the magnitude of the optimal phenotypic change that may be required for persistence in the environment in which a disperser settles, and, hence, whether small or large mutations favor adaptation to the new environmental conditions (Malcom 2011). In order to better understand the factors that drive the distribution and dynamics of species ranges, it is thus crucial to explore these interactive effects and their impact on the species' rates of adaptation to novel conditions.

Here, we investigate the potential co-action among landscape structure, genetic architecture, and dispersal on the rate of adaptation at range margins using an individualbased allelic model (Schiffers and Travis 2014). In particular, our questions are: how are the adaptation rate of a population (and hence the pace of range expansion) and the evolving phenotype distribution affected by 1) landscape structure (smooth gradient landscapes versus patchy landscapes with different grain sizes), 2) the number and effect size of loci coding for adaptation (few loci of large phenotypic effect versus many loci of small effect), 3) differing average dispersal distances and 4) interactive effects among these three factors? To that end, we expose a virtual species to landscapes with locally varying habitat conditions, expressed in the local optimal value for a trait undergoing selection. This trait is governed by several loci, with mutations ensuring trait variability, and with adaptation initially only to one habitat. Adaption can thus evolve to sink environments that are not initially occupied. We analyze the rate of this adaptive process, as well as the degree to which it involves genetic differentiation from the initial source populations.

\section{Methods}

We conducted a series of simulations based on the spatiallyexplicit model framework 'ALADYN' (Schiffers and Travis 
2014, the code is available at <www.katja-schiffers.eu/docs/ Aladyn.zip $>$ ). The model organism in this simulation tool is a hermaphroditic, annual species with a fully outcrossing mating system and discrete, non-overlapping generations. Individuals are diploid, carrying two alleles at each of $L$ unlinked loci, which collectively code for a trait that determines the individual's degree of adaptation to local habitat conditions. Alleles are described by continuous values and are additive within and between loci, i.e. neither epistatic nor pleiotropic effects are considered. An individual's phenotype is directly determined by its genotype, that is, heritability is assumed to be 1 , and there are no environmental effects on the phenotype, either developmental noise or plasticity (Atkins and Travis 2010, North et al. 2011, Phillips 2012).

\section{Model system}

Population dynamics take place upon a grid of 16 by 64 cells. The two long edges of the grid are connected, building a cylinder, to reduce edge effects. Single cells are characterized by a variable that reflects habitat conditions, and in particular, the value of the trait that is optimal for survival in that cell; this optimum by convention ranges between 0 and 2. Density dependence occurs in discrete space with each grid cell supporting several individuals, the maximum number given by the local carrying capacity, $K=5$, which is assumed to be constant across the grid. To facilitate modeling of the dispersal process, individuals are located in continuous space; each individual's location is defined by continuous $\mathrm{x}-\mathrm{y}$ coordinates ranging from 0.0 to 16.0 and 0.0 to 64.0 , respectively. Use of discrete space to model density-dependent processes and continuous space to model dispersal has considerable advantages in terms of computational speed whilst avoiding potential artifacts that arise when continuous dispersal kernels are discretized (Dytham and Travis 2006, Bocedi et al. 2012).

\section{Life cycle}

Within each generation, the following processes are simulated in sequential order: 1) gametes are produced, and at this time recombination and mutations occur; 2 ) male gametes disperse and fertilize ovules, and all adults die after reproduction; 3) offspring disperse; 4) density-independent selection modulates the survival probabilities of the juveniles; and finally, 5) density-dependent mortality is imposed on the surviving offspring. Stochasticity is present in several places across the life history, including individual birth and death events, mutation, genetic drift, gene flow, and selection.

\section{1) Gamete production}

All individuals that survive to adulthood produce male and female gametes, and can thus potentially bear offspring. The number of ovules produced by each individual is Poisson distributed with average $R$, which is assumed to be independent of the individuals' fit to the local optimum (all parameter values are provided in Table 1). Male gametes are only produced when an individual has been randomly chosen as a mating partner (see below); their number is assumed to not be limiting. At the time of gamete production, alleles mutate at rate $\mu$; each mutation is considered unique. In this infinite alleles model, the mutational effect, i.e. the amount by which the allelic value is changed, is drawn from a zero-mean normal distribution with variance $\alpha^{2}$. Loci recombine freely without linkage.

\section{2) Gamete dispersal and encounter}

Male gamete dispersal is characterized by a log-normal, isotropic dispersal kernel (Greene et al. 2004), with average $\log$-scale distance $d_{g}$ and a log-scale standard deviation of 0.5 . To gain computational efficiency, gamete dispersal is simulated in a simplified way compared to offspring dispersal (see below) according to the following algorithm: 1) for each ovule, the grid cell from which the mating partner is drawn is determined, by stochastically choosing $\mathrm{x} / \mathrm{y}$ coordinates in the neighborhood of the focal individual, following the gamete dispersal kernel. 2) The mating partner is then randomly drawn from all individuals inhabiting the respective grid cell, excluding the focal individual as outcrossing is obligatory. 3) In case an empty grid cell is chosen in the first place, the procedure is repeated up to 99 times. 4) If all trials are unsuccessful, the ovule remains unfertilized. As this is more likely for isolated individuals with no neighbors, weak Allee effects might occur. This approach does not account for the fact that the probability for each patch to provide a gamete depends on the local density of individuals, i.e. an ovule may be more likely to be fertilized by a gamete from a far but densely populated region than from a close but sparsely populated one. To test for undesirable effects of this simplification, we also developed an implementation of gamete dispersal being

Table 1. Parameter values for simulation runs.

\begin{tabular}{lll}
\hline Parameter & \multicolumn{1}{c}{ Description } & \multicolumn{1}{c}{ Values } \\
\hline$R$ & Mean number of offspring per mother individual per generation & 100 \\
$L$ & Number of loci & $4,8,16,32\left(d_{0}\right.$ fixed to 1.6) \\
$\mu$ & Mutation rate per locus per generation & $10^{-7}$ \\
$\alpha^{2}$ & Variance of mutational effect & $2,1,0.5,0.25$ (varying with $L$, see Eq. 2$)$ \\
$\omega^{2}$ & Selection strength & 0.2 \\
$d_{0}$ & Mean dispersal distance offspring (cells) & $0.8,1.6,3.2,6.4(L$ fixed to 16) \\
$d_{g}$ & Mean dispersal distance gametes (cells) & 1.6 \\
$g r$ & Grain size of checkerboard (cells) & $1,2,3,4$ \\
$g l$ & Gradient length (fraction of total landscape length) & $0,0.05,0.1,0.2,0.4,0.6$ \\
$K$ & Carrying capacity per grid cell & 5 \\
\hline
\end{tabular}


more precise in the sense of linking fertilization probability to the exact distance between individuals and considering the effect of the local density of potential mating partners. Comparison between the two approaches showed that there are no obvious differences at the level of evolutionary or demographic dynamics. We thus chose the former, computationally much less intensive method. After reproduction all adults die.

\section{3) Offspring dispersal}

Like gamete dispersal, offspring dispersal is characterized by a log-normal, isotropic dispersal kernel, with average distance $d_{o}$ on the logarithmic scale and standard deviation 0.5 . Individuals falling off the cylinder (approximately 0.5 to $5.5 \%$ of the offspring depending on dispersal distance) are excluded from subsequent simulation dynamics.

\section{4) Selection}

Selection acts on population demography by modulating juvenile survival probability. Individual survival probability $W$ is a function of the squared difference between the individual's phenotype $z$ and the optimal phenotype under the local environmental conditions $\theta(x, y)$. This function follows a normal distribution with maximum 1 and variance $\omega^{2}$ :

$W(z)=\exp \left[-\frac{(z-\theta)^{2}}{2 \omega^{2}}\right]$

The quantity $\omega^{2}$ can be interpreted as the inverse of selection strength of the environment on the individuals, or alternatively as the niche breadth of the species (Roughgarden 1972).

\section{5) Density-dependent mortality}

Local population regulation occurs after selection, acting on the survivors in a grid cell. We assume a simple ceiling form of density dependence: whenever the number of individuals $N$ within a grid cell exceeds its carrying capacity $K$, all $N$ resident individuals are subject to density-dependent mortality with probability of mortality equaling $1-K / N$. For example, if $N=3 K$, each individual dies with a probability of $1-1 / 3=2 / 3$ reducing $3 K$ individuals on average to $K$ individuals. This introduces another realistic dimension of stochasticity to the model, since there will be chance fluctuations in the number of individuals surviving density-dependence.

\section{Simulations}

Simulations were used to test the interactive effects of landscape structure, genetic architecture and average dispersal distances on population growth, the rate of adaptation and intraspecific niche differentiation, i.e. local adaptation (Table 1). For each of the 70 parameter combinations, we ran 50 replicates of 500 generations.

\section{Initialization}

Initially, the environmentally homogeneous left quarter of the landscape $(\theta=0)$ was filled with individuals with mean phenotype $\bar{z}=0$ and population density reaching $K$ (thus with 1280 individuals). Standing phenotypic variation was assumed to be 0.01 (approximately the value that we found to evolve from the mutation-selection balance over 5000 simulated generations). All cells outside of this region were unoccupied.

\section{Landscape structure}

Two different types of landscapes were simulated. In both cases, the left quarter of the grid was assigned habitat conditions of value of 0 and the right quarter a value of 2 . In between was either a continuous gradient of conditions with adjustable length $g l$ ranging from 0 to 0.6 (the proportion of the grid that the gradient spans), or a checkerboard pattern of blocks with variable grain size $g s$ and habitat conditions of 0 and 2, respectively (Fig. 1 and Table 1). That way both the effect of the coarseness, i.e. the abruptness of change in environmental values across space, as well as the effect of the grain of the pattern could be tested.

\section{Genetic architecture}

The genetic architecture underlying adaptation was modified by varying the number of loci $L$ from 4 to 32 (Table 1 ). To keep the mutational input per generation $V_{m}$ constant, the variance $\alpha^{2}$ of the normal distribution from which mutational effects were drawn was adapted as follows:

$\alpha^{2}=V_{m} /(2 L \mu)$

Since the mutation rate per locus $\mu$ was held constant, varying the number of loci $L$ amounted to changing mutational effects from few mutations with typically large phenotypic effects to many mutations with small effects.

\section{Dispersal distance}

Offspring dispersal distances $d_{0}$ were varied between 0.8 grid cell units and 6.4 grid cell units (Table 1). The average
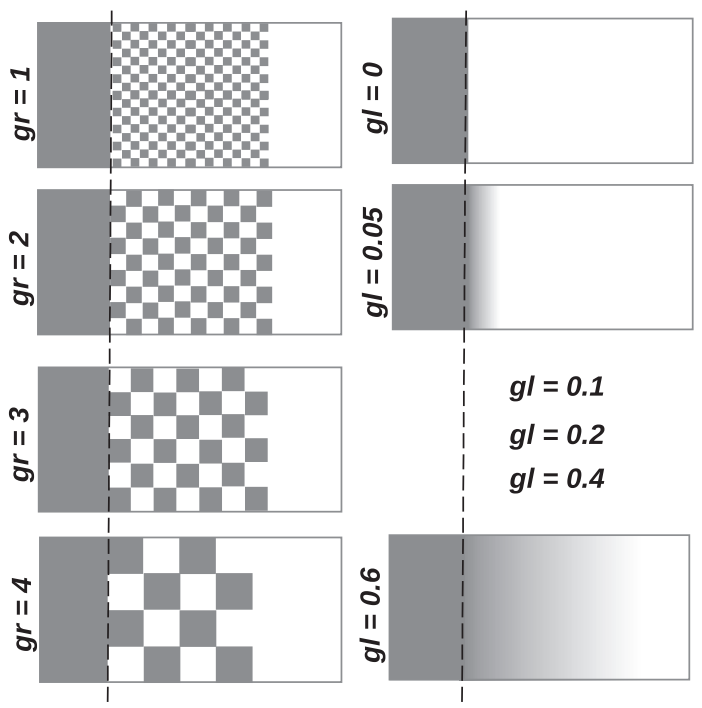

Figure 1. Simulated landscape structures with dark gray area indicating habitat where $\theta=0$, and white areas habitat where $\theta=2$. Habitat conditions reflect the value of the trait that is optimal for survival at that location. Left column: checkerboard patterns with varying grain size. Right column: gradients of environmental conditions with different lengths and slopes. 
gamete dispersal distance $d_{g}$ was held constant to facilitate interpretation of the results. We chose to apply a dispersal kernel for gamete dispersal even though we did not test for effects of different dispersal distances (instead of e.g. randomly drawing individuals from the same patch), to allow for stochasticity in the distance of mating partners and to reduce unrealistic Allee effects particularly at the range boarder, where patches may be frequently inhabited by single individuals.

\section{Estimating the rate of adaptation}

We estimated the rate of adaptation as the change over time of the phenotypic value of those individuals in each generation that were best adapted to the novel environmental conditions $(\theta=2)$ :

$r_{a}=\left(X_{2}-X_{1}\right) /\left(T_{2}-T_{1}\right)$

where $X_{1}$ and $X_{2}$ are phenotypic values averaged across the 30 best adapted individuals and across replicates at sequential time points $T_{1}$ and $T_{2}$, respectively. $T_{1}$ represents the first simulated generation and $T_{2}$ the generation at which the average phenotypic value reaches for the first time its maximum level of adaptation, calculated as the average over the last 20 simulated time steps.

As we base the calculation of the rate of adaptation on the average phenotypes across replicates (rather than first estimating the rate of adaptation for each replicate and then averaging, which does not reflect the true average rate), standard errors were estimated using 99 bootstrap samples from the 50 replicate runs.

\section{Results}

In the absence of evolutionary processes (no mutation and no recombination allowed in the simulations), range sizes increased up to the point where the population filled all suitable habitat. In the case of checkerboard landscapes, this was all habitat of condition $\theta=0$, hence $50 \%$ of the total area and an average population size of 2560 individuals. In gradient landscapes, the proportion of suitable habitat was higher for longer gradients and average population sizes averaged across all genetic architectures and dispersal distances ranged from 1360 individuals for $g l=0$ to 3732 individuals for $g l=0.6$. After the initial increase in population size, further growth was hampered by adverse natural selection so that the remaining areas of the grid remained empty. In the following, we report results based on the total population growth aggregated across the landscape, resulting from the combined effects of demographic and evolutionary dynamics over 500 generations. This number of generations did not always allow equilibrium to be reached, but was sufficient to calculate a diagnostically conclusive estimate for the rate of adaptation (Supplementary material Appendix 1, Table A1).

Overall, we found that the rate of adaptation and hence the speed of range expansion depended strongly on landscape structure, genetic architecture, and dispersal ability. We also found that these factors interact in their effects on genetic and demographic dynamics. Likewise, the phenotypic structure of the simulated populations varied markedly among scenarios.

\section{Effects of landscape-structure on evolutionary and demographic dynamics}

In checkerboard landscapes, the grain of the spatial pattern had no clear effect on the rate of adaptation (Fig. 2a). In comparison, in gradient landscapes, adaptation rate showed a clearer response to the length of the gradient: longer gradients had a positive effect on the rate of adaptation until an intermediate value of $g l=0.2$; for longer and shallower gradients, adaptation rate decreased again (Fig. 2c, d). As expected, these results were reflected by population growth rates. Grain size of checkerboard landscapes affected population growth only marginally (Fig. 3a, i-iv), while populations grew distinctly more quickly on landscapes with gradients of a length of $g l=0.2$ than of $g l=0.05$ (Fig. 3b, i-iv).

Landscape structure also impacted intraspecific niche differentiation. After 500 generations, for most parameter combinations, two locally adapted and distinct phenotypes had evolved in checkerboard landscapes (Fig. 4a, Supplementary material Appendix 1, Fig. A1 and A3). For highly dispersive species, however, very fine-grained patterns also allowed for the evolution of an additional, intermediate phenotype (Fig. $4 \mathrm{~b}$ and Supplementary material Appendix 1, Fig. A1a). In gradient landscapes, the full range of phenotypes only evolved on long, shallow gradients and when dispersal distances were large (Fig. 4c, Supplementary material Appendix 1, Fig. A2c, d). Still after 500 generations, adaptation showed a slight spatial 'lag', with phenotypes not fully matching the local optimum (Fig. 4c). On steeper gradients, only one intermediate type established besides the ones adapted to the extremes of the gradient (Fig. 4d).

\section{Effects of genetic architecture on evolutionary and demographic dynamics}

In checkerboard landscapes, an increasing number of coding loci generally had a negative effect on the rate of adaptation and population growth (Fig. $2 \mathrm{~b}$ and $3 \mathrm{a}$, v-viii). For an intermediate number of loci (and thus mutational effect size), adaptation and range expansion were possible, but proceeded slowly in comparison to rates obtained for genetic architectures of few loci of large effect (Fig. 3a, v-viii). This effect was reversed for gradient landscapes. Here, architectures of many loci with small phenotypic effect sizes resulted in the highest rates of adaptation and population growth (Fig. $2 \mathrm{~d}$ and $3 \mathrm{~b}$, v-viii). This pattern was more pronounced for intermediate gradient lengths than for very short or long ones (Fig. 2d).

Apart from the fact that for genetic architectures with many loci adaptation in checkerboard landscapes was often not possible, the number of loci did not have a large impact on the overall distribution of phenotypes (Supplementary material Appendix 1, Fig. A3-A4). It did, however, affect 
Checkerboard pattern
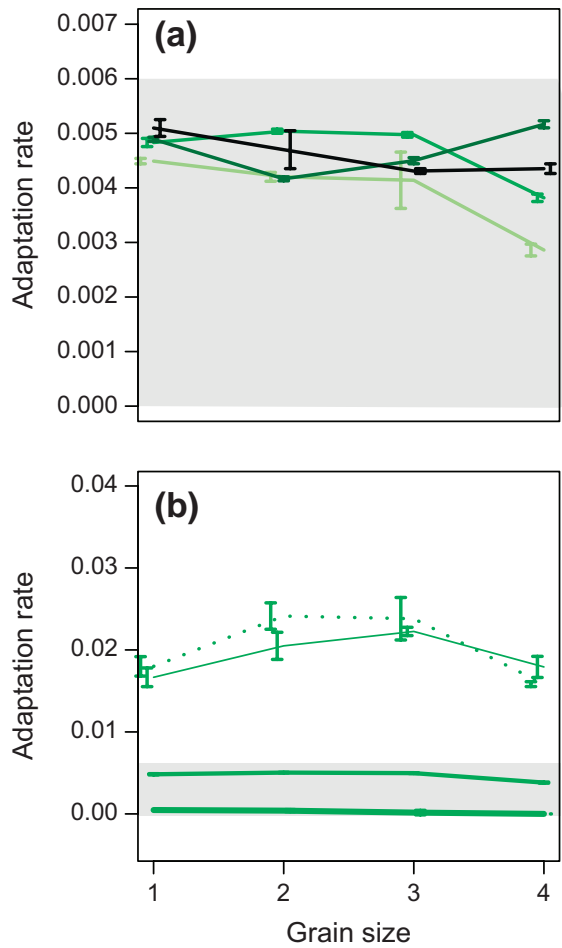

Gradient landscape
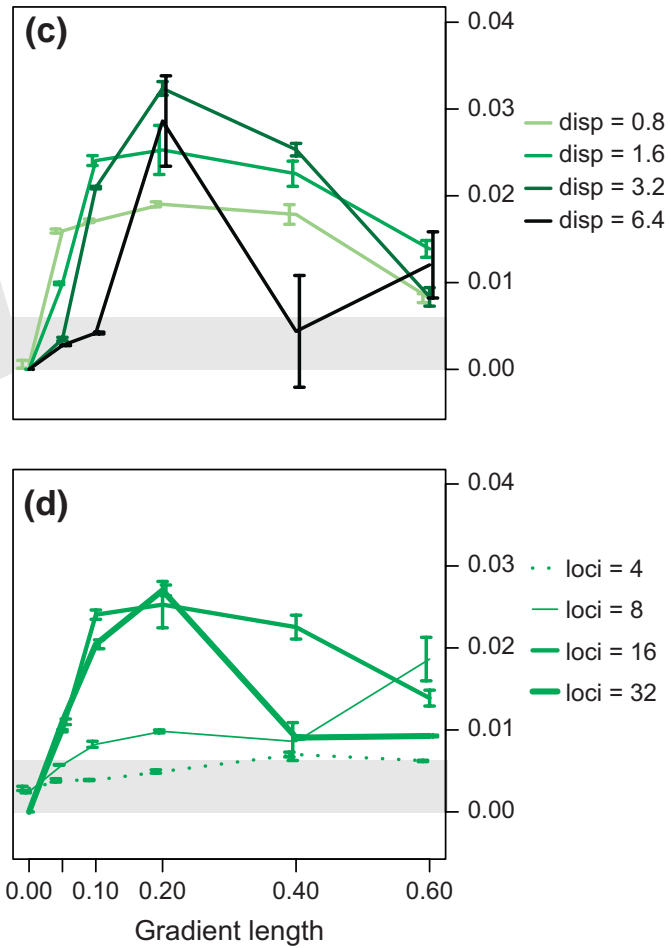

Figure 2. Rate of adaptation in response to grain size of checkerboard patterns (a and b) and to gradient length (c and d). Upper panels ( $\mathrm{a}$ and c) compare scenarios of different dispersal distances indicated by line color and a fixed genetic architecture with $L=16$, lower panels ( $\mathrm{b}$ and $\mathrm{d}$ ) scenarios of different genetic architectures indicated by line type and a fixed dispersal distance of $d_{0}=1.6$. Depicted values are averages over 50 replicate runs, error bars indicate standard errors ( $\pm 1 \mathrm{SE}$ ) estimated from 99 bootstrap samples. Note the different scales of the y-axes indicated by gray polygons.

the level of remaining maladaptation in the new habitat, i.e. the average distance of a phenotype to the locally optimal phenotype (Fig. 5, Supplementary material Appendix 1, Fig. A3-A4). Overall, genetic architectures with many loci of small effects resulted in better adaptation to the new habitat, when landscape structure was not too coarse. This pattern was apparent for scenarios of intermediate to long gradients (Fig. 5b), but was even more pronounced for fine-grained checkerboard patterns (Fig. 5a), where this type of architecture typically hampered the probability and rate of adaptation. Only for large grain size did genetic architectures of 32 loci lead to higher levels of maladaptation.

\section{Effects of dispersal on evolutionary and demographic dynamics}

Dispersal distance had only a weak effect on the rate of adaptation in checkerboard landscapes (Fig. 2a). In gradient landscapes, differences in adaptation rates between dispersal distances were much larger (Fig. 2c, note the different scales of the y-axes), but showed no consistent pattern across gradient lengths (see also paragraph below). Phenotype distribution depended strongly on dispersal. Only for the largest dispersal distance was the evolution of phenotypes different from those adapted to the habitat extremes possible (Supplementary material Appendix 1, Fig. A1-A2).

\section{Interactive effects among landscape-structure, genetic architecture and dispersal}

Several interactive effects on the rate of adaptation and population growth occurred among landscape-structure, genetic architecture and dispersal. Generally, adaptation rate in the two landscape types responded differently to genetic architecture and dispersal distance (Fig. 2). While in checkerboard systems few loci with large effects clearly favored rapid adaptation, the opposite was true for gradient landscapes. Also, adaptation rates in gradient landscapes depended strongly on dispersal distance, which was not the case for checkerboard systems (Fig. 2). In addition, we observe a clear pattern of an interactive effect between gradient length and dispersal distance in gradient landscapes: shorter dispersal distances were optimal for shorter gradients and longer distances maximized maximized the rate of adaptation in landscapes of intermediate to long gradients (Fig. 2c).

\section{Discussion}

Using an individual-based simulation model, we have explored the dynamics of evolutionary adaptation of a trait as a joint function of its genetic architecture, the average dispersal distance of the considered species and the spatially explicit landscape settings of the area the population 


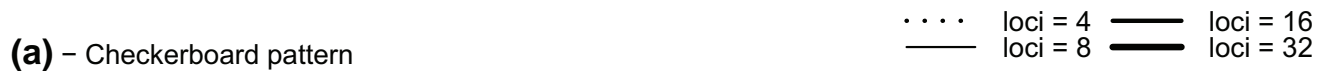

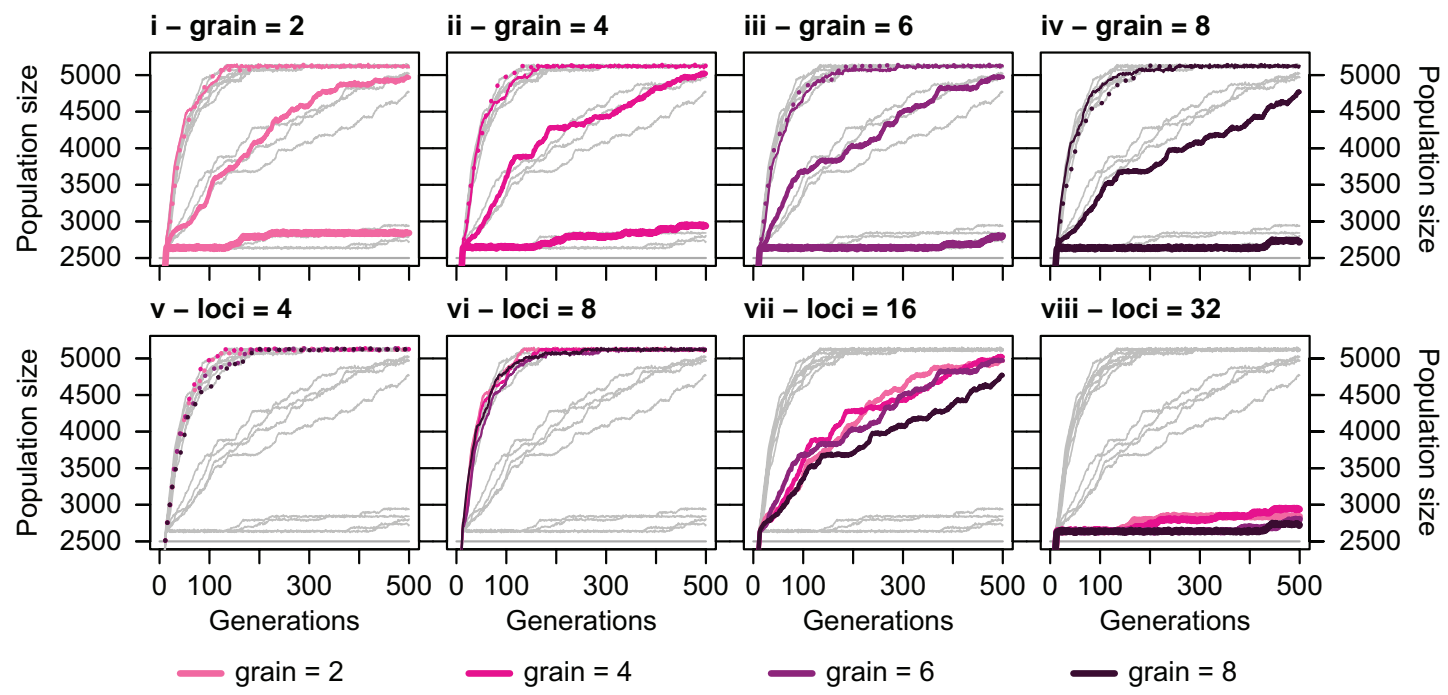

(b) - Gradient landscape
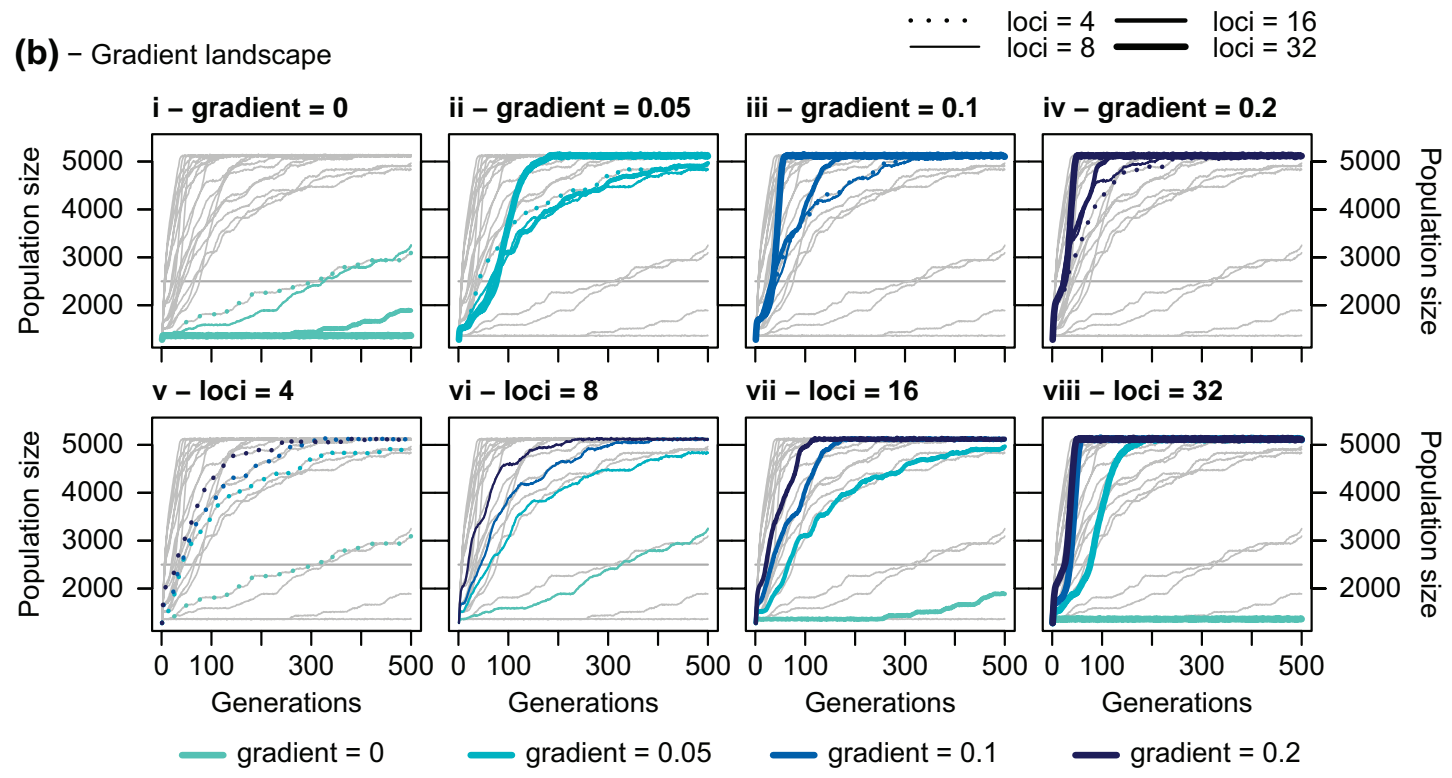

Figure 3. Population size (combined over the entire landscape) over time for checkerboard patterns (a) and gradient landscapes (b). In each panel i-viii, population dynamics for all parameter combinations of genetic architecture and landscape structure are shown as gray lines. Within each panel, results are highlighted by colored lines for one parameter value fixed - either for landscape structure indicated by line color, or for genetic architecture indicated by line type. For example, panel (a) $-\mathrm{i}$ depicts results for grain size $=2$ (indicated by light pink) and different number of loci; panel (a) - v depicts results for 4 loci (indicated by dotted lines) and different grain sizes. For each scenario, dispersal distance was fixed to $d_{0}=1.6$ and 50 replicates were run.

inhabits. In congruence with earlier studies of range evolution along gradients, adaptation and population growth tended to be quicker on intermediate to long gradients (Kirkpatrick and Barton 1997). Dispersal distances and genetic architecture showed clear effects on the adaptive and demographic dynamics of the populations (reminiscent, for instance, of effects shown by Bridle et al. [2010] and Holt and Barfield [2011]). More striking, though, is the highly non-linear interplay that arose among these factors, in particular between landscape structure and the genetic architecture of traits.

\section{Landscape structure and genetic architecture jointly affect rates of adaptation}

At first sight, the interactions among landscape structure, the coarseness of the landscape and the genetic architecture of the traits under selection appear complex. On close inspection, though, we suggest that they are traceable to some fundamental evolutionary mechanisms contained in the model.

The main factors determining the probability and rate of adaptation are population size, the standing genetic variation of the traits under selection, mutation rate and the 

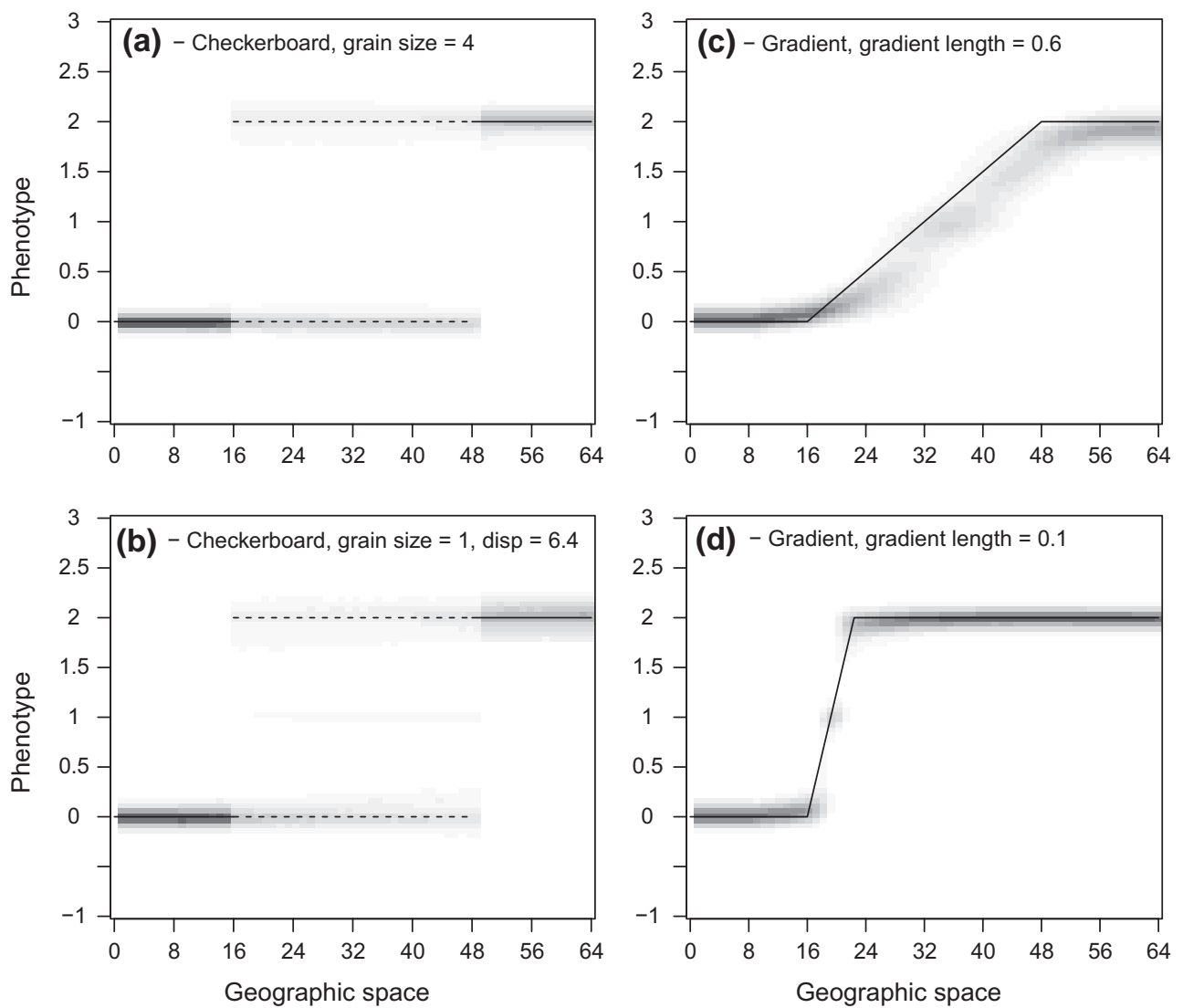

Figure 4. Phenotype distribution after 500 simulated generations, averaged over all runs with $L=16$ and a given spatial structure. (a) Checkerboard pattern of grain size 4 and dispersal distance $d_{o}=1.6$, (b) checkerboard pattern of grain size 1 and dispersal distance $d_{0}=6.4$ (c) gradient landscape of gradient length $=0.6$ and dispersal distance $d_{0}=1.6$, and (d) gradient length $=0.1$ and dispersal distance $d_{o}=1.6$. Black lines indicate local phenotypic optima along the long edge of the grid (solid for areas with only one optimum and dashed for areas with two optima).

distance between optima in source and sink habitats (Bürger 2000). We found differences in adaptation rates even though all of the above factors were kept constant. Going back to the basic mechanisms of evolutionary dynamics helps explaining this discrepancy: in very simple terms, when adaptation depends only on mutational input and not on standing genetic variation, it is the absolute number of mutations per generation and the probability of fixation of
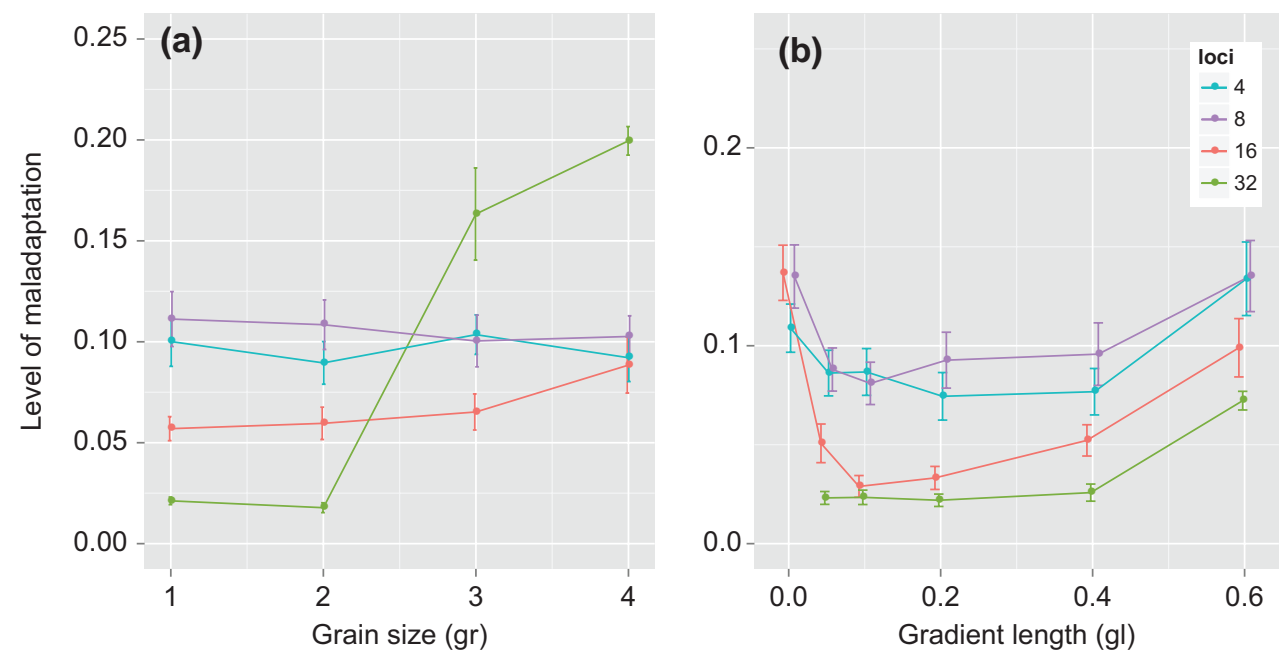

Figure 5. Level of maladaptation (locally optimal phenotype - average realized phenotype) in previously unsuitable habitat $(\theta=2)$ after 500 generations for simulations of checkerboard landscapes (a) and gradient landscapes (b) with $d_{0}=1.6$. Depicted values are averages over 50 replicate runs, error bars show standard errors $( \pm 1 \mathrm{SE})$. Different numbers of loci are indicated by line color. 
these mutations that determine the rate of adaptation. The number of mutations is determined by both population size and mutation rate, but also by the number of loci per individual that potentially mutate. Likewise, fixation probability is a function of population size, the distance of optima between habitats, and also the effect size of mutations (Behrman and Kirkpatrick 2011). Thus, for instance, the harsher the conditions in the sink habitat, as measured by the decrease in survival probability in our model, the greater the effect size of a mutation must be on the phenotype to not get lost due to selection (Kawecki 2008, Holt and Barfield 2011). In other words, the coarseness of the genetic architecture has to match that of the environmental variation (see also Malcom 2011). In our model system, however, we assume that number and effect size of mutations are inversely correlated, leading to a potential trade-off between number and phenotypic effect of mutations. Against this conceptual background, the observed results can be interpreted straightforwardly.

In landscapes of checkerboard-like structure or, more generally, patchy structures with two distinct habitat types, the distance between phenotypic optima is unique and relatively large. Thus, mutations that could improve survival probability in the novel habitat need to have large phenotypic effects to be able to persist (Kawecki 2008, Holt and Barfield 2011), so that the positive effect of genetic architectures with few loci of large effects outweighs that of many loci with small effects. This finding does not vary with checkerboard grain-size. In gradient landscapes, however, the optimal effect size depends on the steepness of the gradient: on very short and steep gradients, the rate of adaptation and population growth is optimal for large mutations, while on shallower gradients it is optimal for smaller mutational effects. Gomulkiewicz et al. (2010) explored how variation in the number of loci influenced persistence of a population colonizing into a harsh, novel environment (in effect a step function, relative to the ancestral environment). When the difference in the initial maladapted and (potential) final adapted state of the population was fixed, in general an increase in the number of loci retarded the rate of adaptation to the novel environment, making extinction more likely than adaptation. In effect, a given amount of selective change was partitioned among more loci, making the selection coefficient at each loci smaller.

Genetic architecture has an additional effect on the match between a mutant's phenotype and the local optimum: smaller mutational steps and recombination allow closer approximation of the phenotypic optimum than do mutations of large effect. The higher precision of adaptation for genetic architectures with many loci with small phenotypic effects was reflected by lower levels of genetic load in our simulations, particularly in gradient landscapes.

\section{Dispersal modulates the apparent coarseness of the landscape structure}

When the phenotype conferring the greatest fitness varies locally due to habitat heterogeneity, the match between the average realized phenotype and local optimum depends not only on the spatial turnover in habitat conditions but also on the average dispersal distance of individuals. The reason is that the coarseness of the landscape has to be gauged by the average dispersal distance of an individual in order to evaluate the expected rate of change in habitat conditions caused by dispersal (Phillips 2012). Gradients, for instance, appear steeper, and checkerboard patterns more fine-grained, for highly dispersive individuals than for individuals with short dispersal distances. Hence, in gradient landscapes, the match between a mutant's phenotype and the local optimum (and therefore the fixation probability of the mutation and the rate of adaptation) depends on the threefold influence of gradient length, mutational effect size, and dispersal distance. A good match to the optimum phenotype can, for instance, arise either from small mutations and short dispersal distances, or from large mutations and long dispersal distances, whereby the steepness of the gradient determines the optimal relationship between dispersal distance and mutational effect. This interactive effect is reflected by the differences in dispersal distances conferring highest adaptation rates, as a function of gradient length (Fig. 2c): given a constant number of loci governing trait values, short dispersal distances maximize adaptation rates for short gradients, while highest rates of adaptation occur for larger dispersal distances on longer gradients.

Nonlinear effects of various factors were also found in some prior studies on evolution at range margins. For instance, in a one-locus model, Gomulkiewicz et al. (1999) found that adaptive evolution in a sink population was facilitated by intermediate dispersal rates (number of immigrants per generation), rather than very low dispersal (which reduces the genetic variation available for selection), or very high dispersal (which leads to negative density dependence and gene swamping). Other frequently observed impacts of dispersal, like a negative effect of large dispersal distances on demographic dynamics as a result of migration load (Alleaume-Benharira et al. 2006), did not occur in our simulations; decreases in population size were effectively counteracted by high reproduction rates. The decrease in adaptation rate at large dispersal distances on gradients of intermediate length could reflect several co-occurring processes. The high number of dispersing zygotes may lead to gene swamping and potentially to 'ecological swamping' by pushing local populations above carrying capacity. Also, high dispersal implies that mutations that are favorable at a given site can be readily lost due to high emigration rates (Holt 2011). As the decrease in adaptation rate could not be detected for longer gradients, however, the more likely reason may be the higher discrepancy of the dispersing individuals phenotypes to the phenotypic optima at sites far away from home locations than to those closer by.

\section{Large dispersal distances and fine-grained landscape structures favor the evolution of intermediate phenotypes}

As a second component, we also investigated intraspecific niche differentiation that arose from the diversifying selection of the different adaptive optima in habitat types (Hedrick 2006). In checkerboard landscapes and on steep gradients, in 
most cases, two distinct phenotypes developed, each adapted to one of the two extreme habitat types (i.e. 0 and 2, respectively). For a few parameter combinations, however, an additional phenotype emerged in the checkerboard landscape that best matched intermediate conditions of value 1 . Due to the fixed niche width in the simulations, an intrinsic functional interference was implied in performing equally well in different habitats, leading to a negative genetic correlation across environments (Futuyma and Moreno 1988) and lower survival probabilities of the intermediate type in the extreme habitats. Still, the intermediate phenotype showed positive population growth and, hence, stable population sizes in habitat conditions 0 and 2 , fulfilling the role of a 'jack-ofall-trades and a master-of-none'.

The occurrence of the intermediate phenotype followed the patterns previously recorded in literature for phenotypic generalists. The evolution of versatile phenotypes were favored by fine-grained variation of habitat conditions (Levins 1968, Kassen 2002) as well as by large dispersal distances (as in Brown and Pavlovic 1992, Holt and Gaines 1992, Kawecki 1995, Holt 1996, Day 2000). This is due to the increased frequency of individuals dispersing among habitat types, which homogenizes allelic frequencies (Ronce and Kirkpatrick 2001).

As phenotypes that were adapted to the extreme habitat conditions were always abundant in our simulations due to the presence of relatively large homogeneous areas of these habitats, our conclusions on the maintenance of phenotypic diversity are generally reversed in comparison to other studies (Kassen 2002). The highest diversity of genotypes across the checkerboard landscape arose when an intermediate phenotype evolved due to large dispersal distances and finegrained landscape structures. Whereas in other studies only generalist phenotypes survived, the structure of our simulated landscapes allowed spatially separated co-occurrence of intermediate phenotypes with those optimally adapted to habitat extremes.

\section{Model limitations and perspectives}

While this study is one of the first to investigate the dynamics of niche evolution and range expansion in complex, continuous landscapes, a number of additional factors that potentially influence rates of adaptation remain to be tested. For example, epistatic and pleiotropic effects can interact with the spatial constellation of habitat in a system of several interconnected patches to affect evolutionary outcomes (Kimbrell 2010). In these cases, complex genetic architectures often lead to genetic canalization, which might hamper adaptation to new environments (Kimbrell 2010). Also, linkage might modulate adaptive dynamics as selection tends to create positive linkage disequilibrium between beneficial alleles, resulting in larger genetic variation and a greater response to selection (Kawecki 2008). Further, while we have investigated the effects of offspring dispersal only, gamete and offspring dispersal might have different, if not contrasting, effects on adaptive dynamics (Alleaume-Benharira et al. 2006, Aguilée et al. 2013). In changing climates, evolutionary dynamics may be altered through changes in spatial patterning of habitat (McInerny et al. 2007) or in the geometry of spatial interactions during range shifts (McInerny et al. 2009). In addition, the effect of dispersal might depend on the order of life-history events, the presence of density dependence during selection and the mode of reproduction of the considered species (Holt and Barfield 2011). We would expect, for instance, that adaptation and range expansion are slower when selection is applied before offspring disperse, as newly arising mutations beneficial for survival in the new environment cannot be efficiently filtered out at that stage. Densitydependent soft selection on the other hand, will probably allow for faster range expansions, since a fraction of individuals will always survive even though potentially not well adapted to environmental conditions in absolute terms. At the same time, stochastically implemented soft selection will probably slow down the rate of adaptation, as it is less efficient in the promotion of beneficial mutations compared to hard selection. All these factors may alter the effect of landscape structure through the interplay of ecological and evolutionary processes as exemplified by dispersal, which comes under strong selection at range margins (Dytham 2009, Phillips 2012). The interplay between local adaptation and dispersal in driving the dynamics of species' ranges is an area requiring further attention (Schiffers et al. 2012).

In this study, we have modeled a single species, which is representative for most existing theory developed to explore the eco-evolutionary dynamics of species ranges. However, there is considerable scope to extend this work to explore the dynamics of species that interact competitively, through predation, parasitism, mutualism, etc. Early theoretical work has demonstrated how inter-specific interactions influence the dynamics of ranges (Brooker et al. 2007, Singer et al. 2013, Svenning et al. 2014), but with a few recent exceptions (Case and Taper 2000, Price and Kirkpatrick 2009, Holt et al. 2011, Bocedi et al. 2012, Norberg et al. 2012), little work has so far explored the interplay between species interactions and local adaptation. In making extensions of this type, it will be interesting to consider how the local co-adaptation between species determines the distribution and dynamics of individual species ranges.

Finally, in our model, adaptation almost completely depended on newly arising mutations, because standing genetic variation was too small to allow adaptation to the sink habitat through recombination. This lack of genetic diversity might seem unlikely, as many traits show a substantial amount of standing genetic variation in natural populations (Houle 1992). Evolutionary dynamics for such traits might differ strongly from those observed here, as the main drivers of the rates of adaptation in our simulations (the total number of mutations and their fixation probabilities) become less important if not irrelevant. As expected, a small additional simulation study showed that adaptation was much quicker when the standing genetic variation of the first generation was higher (Supplementary material Appendix 1, Table A2). However, our results should be valid for ecologically important traits with low genetic variation, such as desiccation and cold resistance in some Drosophila species (Hoffmann et al. 2003, Kellermann et al. 2009), or traits prone to adaptive trade-offs such as flowering time in monkeyflowers (Angert et al. 2008). 


\section{Conclusions}

Under environmental change and increasing habitat fragmentation, the fate of many species will depend on their potential to track their climatic niches in space and to adapt to novel habitat conditions. Understanding how landscape structure interacts with genetic architecture and dispersal characteristics in driving evolutionary adaptation, will help to interpret observed dynamics of niche evolution and range expansions and to predict the future dynamics of species. Here, we show that factors that drive the fixation probability of newly arising beneficial mutations are of particular importance for understanding a species' adaptation potential. Our results 1) highlight that the likelihood and rate of adaptation depends on the match between the coarseness of the genetic architecture and the coarseness of the environment and 2) show that the optimal match between genetic and environmental coarseness is modulated by the dispersal abilities of the considered species.

Available at: <www.katja-schiffers.eu/docs/Aladyn.zip >.

Acknowledgements - This study arose from two workshops entitled 'Advancing concepts and models of species range dynamics: understanding and disentangling processes across scales'. Funding was provided by the Danish Council for Independent Research Natural Sciences (grant no. 10-085056 to S. Normand). KS, SL and WT received funding from the European Research Council under the European Community's Seven Framework Programme FP7/2007-2013 Grant Agreement no. 281422 (TEEMBIO). FMS acknowledges funding from the German Research Foundation (DFG, SCHU-2259/5-1), ROW acknowledges funding from the Swiss National Science Foundation (Synergia Project CRS113125240, Early Postdoc Mobility Grant PBZHP3_147226), and KM (DEB-0919230) and VME (DEB-1256316) acknowledgefunding from the U.S. National Science Foundation. $\mathrm{RDH}$ thanks the Univ. of Florida Foundation.

\section{References}

Aguilée, R. et al. 2013. How does pollen versus seed dispersal affect niche evolution? - Evolution 67: 792-805.

Alleaume-Benharira, M. et al. 2006. Geographical patterns of adaptation within a species' range: interactions between drift and gene flow. - J. Evol. Biol. 19: 203-215.

Angert, A. L. et al. 2008. Using experimental evolution to investigate geographic range limits in monkeyflowers. - Evolution 62: 2660-2675.

Atkins, K. E. and Travis, J. M. J. 2010. Local adaptation and the evolution of species' ranges under climate change. - J. Theor. Biol. 266: 449-457.

Behrman, K. D. and Kirkpatrick, M. 2011. Species range expansion by beneficial mutations. - J. Evol. Biol. 24: 665-675.

Bell, G. and Gonzalez, A. 2011. Adaptation and evolutionary rescue in metapopulations experiencing environmental deterioration. - Science 332: $1327-1330$.

Bocedi, G. et al. 2012. Projecting species' range expansion dynamics: sources of systematic biases when scaling up patterns and processes. - Methods Ecol. Evol. 3: 1008-1018.

Bourguet, D. et al. 2004. Fitness costs of insecticide resistance in natural breeding sites of the mosquito Culex pipiens. - Evolution 58: 128-135.
Bridle, J. and Vines, T. 2007. Limits to evolution at range margins: when and why does adaptation fail? - Trends Ecol. Evol. 22: $140-147$.

Bridle, J. et al. 2010. Why is adaptation prevented at ecological margins? New insights from individual-based simulations. - Ecol. Lett. 13: 485-494.

Brooker, R. W. et al. 2007. Modelling species' range shifts in a changing climate: the impacts of biotic interactions, dispersal distance and the rate of climate change. - J. Theor. Biol. 245: 59-65.

Brown, J. S. and Pavlovic, N. B. 1992. Evolution in heterogeneous environments: effects of migration on habitat specialization. - Evol. Ecol. 6: 360-382.

Bürger, R. 2000. The mathematical theory of selection, recombination, and mutation. - Wiley.

Case, T. and Taper, M. 2000. Interspecific competition, environmental gradients, gene flow, and the coevolution of species' borders. - Am. Nat. 155: 583-605.

Davis, M. B. and Shaw, R. G. 2001. Range shifts and adaptive responses to quaternary climate change. - Science 292: 673-679.

Day, T. 2000. Competition and the effect of spatial resource heterogeneity on evolutionary diversification. - Am. Nat. 155: 790-803.

Dytham, C. 2009. Evolved dispersal strategies at range margins. - Proc. R. Soc. B 276: 1407-1413.

Dytham, C. and Travis, J. 2006. Evolving dispersal and age at death. - Oikos 113: 530-538.

Fisher, R. A. 1930. The genetical theory of natural selection. - Clarendon Press.

Fortin, M.-J. et al. 2005. Species' geographic ranges and distributional limits: pattern analysis and statistical issues. - Oikos 108: 7-17.

Fournier-Level, A. et al. 2011. A map of local adaptation in Arabidopsis thaliana. - Science 334: 86-89.

Frean, M. et al. 2013. The effect of population structure on the rate of evolution. - Proc. R. Soc. B 280: 20130211.

Futuyma, D. and Moreno, G. 1988. The evolution of ecological specialization. - Annu. Rev. Ecol. Syst. 19: 207-233.

Garcia-Ramos, G. and Kirkpatrick, M. 1997. Genetic models of adaptation and gene flow in peripheral populations. - Evolution 51: 21-28.

Gastner, M. T. et al. 2009. Transition from connected to fragmented vegetation across an environmental gradient: scaling laws in ecotone geometry. - Am. Nat. 174: E23-E39.

Gomulkiewicz, R. et al. 1999. The effects of density dependence and immigration on local adaptation and niche evolution in a black-hole sink environment. - Theor. Popul. Biol. 55: 283-296.

Gomulkiewicz, R. et al. 2010. Genetics, adaptation, and invasion in harsh environments. - Evol. Appl. 3: 97-108.

Greene, D. F. et al. 2004. An evaluation of alternative dispersal functions for trees. - J. Ecol. 92: 758-766.

Haldane, J. B. S. 1957. The cost of natural selection. - J. Genet. 55: $511-524$.

Hedrick, P. W. 2006. Genetic polymorphism in heterogeneous environments: the age of genomics. - Annu. Rev. Ecol. Evol. Syst. 37: 67-93.

Hoffmann, A. A. et al. 2003. Low potential for climatic stress adaptation in a rainforest drosophila species. - Science 301: 100-102.

Holt, R. D. 1996. Demographic constraints in evolution: towards unifying the evolutionary theories of senescence and niche conservatism. - Evol. Ecol. 10: 1-11.

Holt, R. D. 2009. Colloquium papers: bringing the Hutchinsonian niche into the 21st century: ecological and evolutionary perspectives. - Proc. Natl Acad. Sci. USA 106: 19659-19665. 
Holt, R. D. 2011. Evolution in source-sink environments: implications for niche conservatism. - In: Liu, J. et al. (eds), Sources, sinks and sustainability. Cambridge Univ. Press, pp. 23-57.

Holt, R. and Gaines, M. 1992. Analysis of adaptation in heterogeneous landscapes - implications for the evolution of fundamental niches. - Evol. Ecol. 6: 433-447.

Holt, R. and Gomulkiewicz, R. 1997. How does immigration influence local adaptation? A reexamination of a familiar paradigm. - Am. Nat. 149: 563-572.

Holt, R. D. and Barfield, M. 2011. Theoretical perspectives on the statics and dynamics of species' borders in patchy environments. - Am. Nat. 178: S6-S25.

Holt, R. D. et al. 2011. Predation and the evolutionary dynamics of species ranges. - Am. Nat. 178: 488-500.

Houle, D. 1992. Comparing evolvability and variability of quantitative traits. - Genetics 130: 195-204.

Hutchinson, G. E. 1978. An introduction to population ecology. - Yale Univ. Press.

Kassen, R. 2002. The experimental evolution of specialists, generalists, and the maintenance of diversity. - J. Evol. Biol. 15: 173-190.

Kawecki, T. J. 1995. Demography of source-sink populations and the evolution of ecological niches. - Evol. Ecol. 9: 38-44.

Kawecki, T. J. 2000. Adaptation to marginal habitats: contrasting influence of the dispersal rate on the fate of alleles with small and large effects. - Proc. R. Soc. B 267: 1315-1320.

Kawecki, T. J. 2008. Adaptation to marginal habitats. - Annu. Rev. Ecol. Evol. Syst. 39: 321-342.

Kellermann, V. et al. 2009. Fundamental evolutionary limits in ecological traits drive drosophila species distributions. - Science 325: 1244-1246.

Kimbrell, T. 2010. Canalization and adaptation in a landscape of sources and sinks. - Evol. Ecol. 24: 891-909.

Kimbrell, T. and Holt, R. D. 2007. Canalization breakdown and evolution in a source-sink system. - Am. Nat. 169: 370-382.

Kirkpatrick, M. and Barton, N. 1997. Evolution of a species' range. - Am. Nat. 150: 1-23.

Kunin, W. E. et al. 2009. Variation at range margins across multiple spatial scales: environmental temperature, population genetics and metabolomic phenotype. - Proc. R. Soc. B 276: 1495-1506.

Lavergne, S. et al. 2010. Biodiversity and climate change: integrating evolutionary and ecological responses of species and communities - Annu. Rev. Ecol. Evol. Syst. 41: 321-350.

Leimu, R. and Fischer, M. 2008. A meta-analysis of local adaptation in plants. - PLoS One 3: e4010.

Levins, R. 1968. Evolution in changing environments: some theoretical explorations. - Princeton Univ. Press.

Supplementary material (Appendix ECOG-00768 at $<$ www.ecography.org/readers/appendix $>$ ). Appendix 1.
Lieberman, E. et al. 2005. Evolutionary dynamics on graphs. - Nature 433: 312-316.

Malcom, J. W. 2011. Smaller gene networks permit longer persistence in fast-changing environments. - PLoS One 6: e14747.

McInerny, G. et al. 2007. Range shifting on a fragmented landscape. - Ecol. Inform. 2: 1-8.

McInerny, G. J. et al. 2009. How range shifts induced by climate change affect neutral evolution. - Proc. R. Soc. B 276: 1527-1534.

Norberg, J. et al. 2012. Eco-evolutionary responses of biodiversity to climate change. - Nat. Clim. Change 2: 747-751.

North, A. et al. 2011. Local adaptation in a changing world: the roles of gene-flow, mutation, and sexual reproduction. - Evolution 65: 79-89.

Orr, H. A. 2005. The genetic theory of adaptation: a brief history. - Nat. Rev. Genet. 6: 119-127.

Phillips, B. L. 2012. Range shift promotes the formation of stable range edges. - J. Biogeogr. 39: 153-161.

Price, T. D. and Kirkpatrick, M. 2009. Evolutionarily stable range limits set by interspecific competition. - Proc. R. Soc. B 276: $1429-1434$.

Pulliam, H. R. 2000. On the relationship between niche and distribution. - Ecol. Lett. 3: 349-361.

Ronce, O. and Kirkpatrick, M. 2001. When sources become sinks: migrational meltdown in heterogeneous habitats. - Evolution 55: $1520-1531$.

Roughgarden, J. 1972. Evolution of niche width. - Am. Nat. 106: 683-718.

Roy, D. B. and Thomas, J. A. 2003. Seasonal variation in the niche, habitat availability and population fluctuations of a Bivoltine thermophilous insect near its range margin. - Oecologia 134: 439-444.

Schiffers, K. and Travis, J. M. J. 2014. ALADYN - a spatially explicit, allelic model for simulating adaptive dynamics. - Ecography 37: 1288-1291.

Schiffers, K. et al. 2012. Limited evolutionary rescue of locally adapted populations facing climate change. - Phil. Trans. R. Soc. B 368: 20120083.

Sexton, J. et al. 2009. Evolution and ecology of species range limits. - Annu. Rev. Ecol. Evol. Syst. 40: 415-436.

Siepielski, A. M. et al. 2013. The spatial patterns of directional phenotypic selection. - Ecol. Lett. 16: 1382-1392.

Singer, A. et al. 2013. Interspecific interactions affect species and community responses to climate shifts. - Oikos 122: 358-366.

Soberón, J. 2007. Grinnellian and Eltonian niches and geographic distributions of species. - Ecol. Lett. 10: 1115-1123.

Svenning, J.-C. et al. 2014. The influence of interspecific interactions on species range expansion rates. - Ecography 37: 1198-1209. 